\title{
Avaliação dos impactos ambientais de um processo industrial utilizando como matéria-prima policarbonatos virgem e reciclado
}

\author{
Evaluation of environmental impacts of an industrial \\ process using virgin and recycled polycarbonate raw material \\ Leornardo de Moura Lacerda' $\mathbb{C}^{\mathbb{D}}$, Andréa Oliveira Nunes ${ }^{2 *}(\mathbb{D}$, \\ Jane Maria Faulstich de Paiva' ${ }^{10}$, Virgínia Aparecida da Silva Moris'
}

口-

\section{RESUMO}

Os impactos ambientais gerados por processos produtivos é um assunto em crescente evidência no mundo organizacional, e a análise comparativa de processos vem sendo utilizada pelas empresas para verificar os impactos ambientais e suas principais fontes de emissões. A análise dos impactos de um processo produtivo, com dois contextos distintos para a produção de hastes de óculos de policarbonato (PC), em uma indústria localizada no interior de São Paulo, foi realizada com a finalidade de comparar os impactos ambientais gerados. Para isso, foram definidos dois cenários: o 1 representa o processo de injeção que utiliza 100\% de PC virgem como matéria-prima, enquanto o 2 usa apenas 20\% de matéria-prima virgem, sendo o restante proveniente de resíduos de PC reciclados internamente. Dados quantitativos e qualitativos referentes aos cenários foram coletados e utilizados para identificar os impactos ambientais gerados com o auxílio da metodologia de avaliação do ciclo de vida (ACV). Com os resultados obtidos e a análise comparativa realizada, foi possível verificar os benefícios ambientais resultantes da reciclagem interna da matéria-prima. A redução dos impactos no cenário 2 em comparação com o 1 foi expressiva, principalmente, devido à diminuição da necessidade da produção de matéria-prima virgem. Em todas as categorias de impactos analisadas, ocorreram reduções - por exemplo, na categoria "mudanças climáticas", a redução foi superior a 70\%.

Palavras-chave: policarbonato; reciclagem; impactos ambientais; avaliação do ciclo de vida.

\begin{abstract}
The environmental impacts generated by production processes is a subject that is increasingly evident in the organizational world and the comparative analysis of processes is an effective method used by companies to verify their environmental impacts and main emission sources. The analysis of environmental impacts for a process with two different scenarios to produce Polycarbonate (PC) glasses temples, in an industry located in the region of São Paulo, was performed in order to compare the environmental impacts generated by both scenarios. Thus, two scenarios were defined: scenario 1 represents the injection process that uses $100 \%$ of virgin PC as raw material; while scenario 2 uses only $20 \%$ of virgin raw material, the remainder PC waste being internally recycled. Quantitative and qualitative data related to the scenarios were collected and used to identify the impacts generated using the life cycle assessment (LCA) methodology. With the results obtained and the comparative analysis performed, it was possible to verify the environmental benefits of using the internal recycling of raw material. The impact reduction in scenario 2, compared to scenario 1 , was significant, mainly due to the decrease of the need to produce virgin raw material. There were no reductions in all impact categories analyzed; for example, reduction was over $70 \%$ in the "climate change" category.
\end{abstract}

Keywords: polycarbonate; recycling; environmental impacts; life cycle assessment.

\section{INTRODUÇÃO}

Com o crescimento populacional e desenvolvimento tecnológico, os produtos à base de polímeros ganharam cada vez mais destaque, resultando em um aumento na produção global de plástico em 500\% nos últimos 30 anos, a qual se espera que continue a crescer, atingindo cerca de 850 milhões de toneladas por ano em 2050 (HUNT et al., 2015). O plástico é um material bastante funcional, de baixos custo e densidade, resistente e que vem sendo usado para substituir materiais 
como cerâmica, madeira e metais. Entretanto, o aumento do consumo de plástico (polímero) é responsável também pelo aumento na quantidade de resíduos sólidos gerados (WANG et al., 2015).

Os resíduos plásticos podem causar problemas ambientais significativos. A maioria dos plásticos não é biodegradável e também não reage quimicamente em ambiente natural. Sendo assim, esses produtos persistem por décadas ou até mesmo durante séculos no meio ambiente. Alguns tipos comuns de plásticos, como o policloreto de polivinila (PVC) e o policarbonato (PC), podem liberar lentamente, em determinadas condições, compostos tóxicos para o ar, a água e o solo (GU; OZBAKKALOGLU, 2016).

Os plásticos são, praticamente, em sua totalidade, produzidos a partir de produtos petroquímicos derivados de petróleo e gás natural. Cerca de $4 \%$ da produção anual de petróleo é utilizada na produção de plásticos, ou seja, o equivalente a 1,3 bilhão de barris por ano (SINGH et al., 2016). Além disso, a produção de plásticos também requer uso de energia, sendo sua fabricação responsável pelo consumo de uma quantidade adicional de combustíveis fósseis, gerando, consequentemente, emissões de gases que geram o efeito estufa (HOPEWELL; DVORAK; KOSIOR, 2009).

Nesse contexto, a reciclagem de resíduos plásticos pode levar à diminuição do uso de matérias-primas virgens; à redução das emissões de dióxidos de carbono, principal gás responsável pelo efeito estufa; e à diminuição na quantidade de resíduos descartados no meio ambiente, proporcionando, assim, uma redução nos impactos ambientais e no esgotamento de recursos naturais (HOPEWELL; DVORAK; KOSIOR, 2009). Zhu et al. (2017) apresentaram os resultados na produção e caracterização de compósitos com PC e fibra de vidro reciclados. Portanto, a reciclagem aparece como uma alternativa no gerenciamento de resíduos, trazendo benefícios econômicos e ambientais.

Segundo Lino, Ismail e Cosso (2013), a redução no consumo de energia por meio da reciclagem pode proporcionar grandes benefícios para o país, já que a produção de material virgem está associada a um grande consumo energético. Em termos gerais, a reciclagem pode ser mais benéfica ao meio ambiente do que métodos tradicionais de produção e gerenciamento de resíduos, como pirólise, tratamentos térmicos e produção de polímeros virgens (GUTIÉRREZ et al., 2016).

Embora a reciclagem proporcione benefícios ambientais e econômicos, uma avaliação do seu desempenho ambiental ainda se faz necessária. Esse processo é considerado ambientalmente apropriado quando seus impactos ambientais são menores do que os gerados na produção de material virgem e do que os causados por alternativas de disposição final, como aterros e incineração (WITIK et al., 2013).

A avaliação do ciclo de vida (ACV) é uma ferramenta de gestão ambiental utilizada para avaliar os impactos ambientais associados a um determinado produto, processo ou serviço em todo o seu ciclo de vida, desde a extração de matérias-primas até a sua disposição final (SETAC, 1993; CHEHEBE, 1997). É possível também comparar matérias-primas e processos mais impactantes e auxiliar na tomada de decisões com o foco na redução dos impactos ambientais. Nos últimos cinco anos, observa-se aumento considerável nas publicações, indicando uma continuidade na tendência da difusão da ACV (ZANGHELINI et al., 2016).

O presente trabalho tem como objetivo comparar os impactos ambientais, usando a metodologia de ACV, de dois cenários diferentes que utilizam como matéria-prima PC virgem e reciclado, para a fabricação de hastes de óculos de segurança/proteção, em uma empresa localizada na região de Sorocaba (SP). No cenário 1, a produção é realizada com $100 \%$ de PC virgem, enquanto no 2 apenas $20 \%$ do PC é virgem, sendo os $80 \%$ restantes do material reciclado internamente na empresa.

\section{REVISÃO DA LITERATURA}

Os polímeros podem ser obtidos por meio de diferentes processos e podem ser empregados em atividades industriais na forma de pellets do material virgem, assim como em misturas mais complexas com outros polímeros ou materiais. A utilização de materiais poliméricos para a produção de componentes que substituem outros materiais em produtos de bem de consumo, como automóveis, tem aumentado consideravelmente na última década. Entretanto, o contínuo aumento de sua utilização em produtos de vida útil curta tem demandado atenção de pesquisadores ao redor do mundo com relação ao aumento da quantidade de resíduos e, como imediata consequência, a geração de impactos ambientais.

A principal alternativa para controlar o impacto ambiental do uso acelerado de materiais poliméricos é o processo de reciclagem dos resíduos gerados. No entanto, um dos maiores desafios no processo de reciclagem é manter a performance do produto produzido com material reciclado, permitindo que este seja utilizado na mesma ou em outra aplicação (DOS SANTOS et al., 2007).

Na última década, tem-se observado demanda crescente pela reciclagem de polímeros descartados, devido principalmente à necessidade de se reduzir a deposição em aterros sanitários e no meio ambiente. Existem muitas opções para a aplicação dos polímeros reciclados, mas a utilização deve ser financeiramente viável, tecnicamente factível e ambientalmente segura, para ser considerada mais do que uma medida temporária e sem grandes benefícios (MERRINGTON, 2011).

De acordo com Al-Sabagh et al. (2016), a reciclagem primária ocorre por meio do processamento de resíduos industriais descontaminados. Esse tipo de reciclagem, muitas vezes, é realizada misturando o material reciclado com o virgem para assegurar qualidade, garantindo as propriedades necessárias ao produto final.

Dois outros métodos para reciclagem de polímeros são os mais amplamente utilizados e estudados: a reciclagem mecânica e a química. 
Esta tem o objetivo de transformar os resíduos sólidos na sua forma primária original e mais simples, como óleos e hidrocarbonetos, para, posteriormente, servirem de matéria-prima para a síntese e fabricação de novos produtos poliméricos. Por outro lado, aquela é um processo físico, no qual o polímero é processado mecanicamente (moagem e extrusão) e, então, é reciclado e utilizado para a produção de um novo componente que pode, ou não, ser utilizado para o mesmo propósito original (HAMAD; KASEEM; DERI, 2013). Existe ainda a reciclagem energética, em que o poder calorífico (teor energético) dos resíduos plásticos é aproveitado por meio da incineração (AL-SABAGH et al., 2016).

A reciclagem mecânica é a mais utilizada na indústria de reciclagem de plásticos, devido a vários fatores, como custo de mão de obra, facilidade e baixo custo de implantação e operação, entre outros. O Instituto Socioambiental dos Plásticos (Plastivida) afirma que o Brasil já está chegando perto do índice de reciclagem mecânica da Europa, que gira em torno de 22\% (SPINACÉ; PAOLI, 2005).

No Brasil, são reciclados, por mês, cerca de 85 mil toneladas de resíduos, incluindo papéis e papelão, polímeros, metais ferrosos e vidro, e a parcela referente aos polímeros atinge um valor de $25.418,3$ toneladas por mês, o que gera uma economia líquida de energia primária igual a 1,21 GW. Toda essa energia economizada está atrelada a uma redução nas emissões de $\mathrm{CO}_{2}$, por conta da substituição da produção de materiais virgens por reciclados.

Essa diminuição pode chegar ao valor de aproximadamente $10 \mathrm{tCO}_{2} \cdot \mathrm{t}^{-1}$ de material, considerando todos os tipos de materiais, sendo os polímeros responsáveis por $4,1 \mathrm{tCO}_{2} \cdot \mathrm{t}^{-1}$ polímero, representando uma grande parcela dessa redução total. $\mathrm{O}$ decrescimento, que pode ser considerado significativo, mostra a influência da reciclagem de polímeros nas emissões e com relação aos ganhos energéticos, principalmente devido à queda na demanda de matérias-primas virgens e também nas operações de transporte (LINO; ISMAIL; COSSO, 2013).

Um dos processos que pode utilizar matéria-prima reciclada para a fabricação de produtos, em algumas aplicações, é a moldagem por injeção. Muito utilizado na conformação de polímeros, esse tipo de processo é um dos métodos mais baratos e eficazes para a produção de grandes volumes de produtos poliméricos, sendo o processo composto de quatro estágios principais: secagem; mistura e dosagem; injeção; e resfriamento e solidificação para a retirada das peças (MADAN et al., 2015).

Segundo Savage e Hartley (2010), a utilização de misturas de polímeros em processos de injeção é de certa maneira simples, pois o material reciclado flui de forma adequada e se incorpora facilmente ao polímero virgem. Podem-se também utilizar diferentes tipos de polímeros juntos, compatíveis, utilizando um moinho, equipamento comum em instalações industriais.

A mistura de polímeros reciclados aos virgens pode melhorar algumas propriedades mecânicas do material final. O estudo de Hamad, Kaseem e Deri, 2013 (2013) mostrou que a combinação de PC reciclado com os materiais virgens, como o copolímero acrilonitrila-butadieno-estireno (ABS), o poli(tereftalato de etileno) (PET) e o ooli(tereftalato de butileno) (PBT), apresentaram resultados satisfatórios em relação à conservação das propriedades mecânicas.

Kozderka et al. (2016) compararam as propriedades mecânicas e os impactos ambientais gerados pelo processamento de polipropilenos (PP) virgem e reciclado. O trabalho mostrou que uma mistura de 50\% de $\mathrm{PP}$ reciclado com $50 \%$ de virgem não interfere de forma significativa nas propriedades mecânicas do produto final, uma vez que o virgem atua como suporte para manter as propriedades mecânicas. Também foi constatado que a mistura apresenta um resultado positivo na redução dos impactos ambientais gerados, quando comparado com o processo utilizando apenas material virgem. A maior parte dessa melhoria está relacionada à redução da produção desse material.

Existem ferramentas que auxiliam a análise da viabilidade ou não da utilização de materiais reciclados, focadas nos impactos ambientais potenciais. A ACV é uma metodologia amplamente utilizada para avaliar os impactos ambientais potenciais existentes durante o ciclo de vida de um produto, processo ou serviço, incluindo, então, as fases de extração e processamento de matérias-primas, fabricação, transporte, distribuição, uso, manutenção, reciclagem, reutilização e disposição final (SETAC, 1993).

A ACV é, pois, uma análise sistemática que consiste em identificar e quantificar os fluxos de materiais e energia ao longo do ciclo de vida, que serão traduzidos em termos de contribuições para as diferentes categorias de impactos ambientais, como exaustão de recursos não renováveis, mudanças climáticas, toxicidade humana, ecotoxicidade $\mathrm{e}$ acidificação. A avaliação comparativa de diversos ciclos de vida concorrentes, com diferentes características, em cenários distintos, é um dos objetivos clássicos tratados na realização de uma ACV. Uma aplicação de tal abordagem de ACV pode ser, por exemplo, a diferenciação entre os impactos ambientais do produto utilizando matéria-prima virgem com materiais reciclados em sua composição (CAMPOLINA et al., 2017).

A utilização de softwares facilita a gestão dos dados coletados para a avaliação de diferentes cenários de ciclo de vida, por meio de um vasto banco de dados (PERCHENART; ROQUESALANE, 2014). Esses softwares possibilitam ainda a compilação dos dados e a quantificação em termos de impactos ambientais; comparações do ciclo de vida de produtos e processos; e análise dos impactos ambientais e financeiros de todas as atividades do sistema. Atualmente, existem vários softwares disponíveis para realização de ACV, como o SimaPro, GaBi e Umberto. Cada um deles disponibiliza também diferentes métodos de avaliação de impacto (CML-IA Baseline 2001, IMPACT 2002+, ILCD 2011, ReCiPe) (CURRAN, 2006; USEPA, 2017).

Nesse contexto, Kozderka et al. (2016) utilizaram a ACV, com o auxílio do software $\mathrm{GaBi}$, em um estudo com o objetivo de analisar os impactos ambientais gerados utilizando PP virgem e reciclado em 
diferentes cenários, considerando diferentes proporções de material reciclado. Ficou evidente que quanto maior a percentagem de material reciclado, menores foram os impactos ambientais gerados.

Em outro estudo, Kua e Lu (2016), por meio da ACV, avaliaram os impactos ambientais da utilização de vidro temperado ou de PC em janelas, com o objetivo de verificar a viabilidade ambiental da substituição do vidro por esse material. O PC é um dos materiais que vem ganhando grande importância na engenharia e está sendo bastante utilizado para substituir o vidro por causa das suas excelentes propriedades mecânicas, leveza e alta transparência. Os autores consideraram para a realização da $\mathrm{ACV}$, para o vidro temperado, as seguintes etapas: extração de matéria-prima, preparo, queima, polimento com fogo, recozimento, corte, transportes interno e externo, têmpera e reciclagem. Por outro lado, o processo de fabricação do PC é bastante complexo e inclui as etapas da produção do petróleo bruto até a produção final de PC. O processo detalhado é apresentado em PlasticsEurope (2011).

De acordo com os resultados obtidos por Kua e Lu (2016), o vidro temperado apresentou impactos superiores ao PC para algumas categorias. No entanto, os valores para o potencial de aquecimento global e ecotoxidade marinha para ambos os materiais se sobrepõe, tornando-se difícil de concluir qual dos materiais é melhor nessas duas categorias de impactos. Considerando o PC, o potencial de aquecimento global foi influenciado majoritariamente pelos processos de extração de matérias-primas (petróleo bruto e gás natural) e os processos combinados de produção de $\mathrm{PC}$, enquanto o potencial de ecotoxicidade marinha tem forte contribuição no processo de reciclagem, evidenciando uma demanda por melhorias no método de reciclagem utilizado. No trabalho, foi constatado que a produção de $1 \mathrm{~kg}$ de PC virgem gera 5,58 kg de $\mathrm{CO}_{2 e q}$. Por outro lado, a reciclagem de $1 \mathrm{~kg}$ de PC, que requer um consumo energético de 0,234 MJ, tem potencial de aquecimento global de apenas $50 \mathrm{~g}$ de $\mathrm{CO}_{2 e q}(0,89 \%$ em comparação ao potencial de aquecimento global do PC virgem). A reciclagem mecânica do PC, considerada nesse estudo, envolve as etapas de granulação, flotação, pré-lavagem, secagem e separação, finalizando com a extrusão do material.

\section{METODOLOGIA}

O trabalho tem como base um estudo de caso em uma empresa localizada na cidade de Sorocaba que atua na área industrial, especialista em operações e processos produtivos, sendo considerada uma grande processadora de polímeros para a produção de produtos utilizados em diversas áreas. Mais especificamente, o estudo avaliou os impactos ambientais do processo de produção de hastes de PC para óculos de segurança, considerando dois cenários distintos a serem comparados. Para isso, foi utilizada a metodologia de ACV, estruturada e normatizada pela norma ISO 14040, que consiste na realização de quatro etapas: definição do objetivo e escopo; análise de inventário; avaliação dos impactos; e interpretação (ISO, 2006).

\section{Objetivo e escopo}

O objetivo deste estudo é comparar os impactos ambientais do processo de produção de hastes de óculos de segurança que utilizam PC como matéria-prima em dois cenários diferentes: no cenário 1, a produção é realizada com $100 \%$ de PC virgem, enquanto no 2 apenas $20 \%$ do PC utilizado é virgem, sendo os $80 \%$ restantes reciclados.

Portanto, foi escolhida, como base de comparação dos dois cenários, a unidade funcional, definida como a produção de um par de hastes de óculos. Todos os dados coletados e quantificações para os fluxos de entrada e saída dos dois cenários foram normalizados para a unidade funcional.

As características do processo foram identificadas e os dados necessários, coletados. Para tal, foram realizadas visitas in loco à empresa. O processo utiliza a moldagem por injeção para conformar o PC na forma do produto desejado. Para preencher as cavidades do molde, a matéria-prima é aquecida e, então, injetada sob pressão por meio de um bico injetor (Sprue) para dentro do molde, os produtos são formados e um resíduo é gerado, o galho de injeção.

Os dois cenários foram identificados e definidos de acordo com as características apresentadas em cada caso. $\mathrm{O} 1$ representa o processo utilizando 100\% de PC virgem para realizar a injeção das hastes plásticas. O processo começa com o recebimento de $100 \%$ do material necessário para a produção, em forma de pellets, proveniente de um fornecedor localizado em Campinas (SP). Por meio do modal viário, a matéria-prima chega até a fábrica e é colocada em um equipamento, localizado ao lado da injetora, chamado desumidificador, que é responsável por eliminar qualquer resíduo de água existente nos pellets que possa prejudicar o processo. Após a desumidificação, o material é enviado à injetora e moldado no formato desejado. No fim do processo, as hastes são separadas manualmente dos galhos de injeção, que são considerados resíduos do processo e não possuem valor agregado algum nesse cenário. As hastes são estocadas para serem fornecidas aos clientes, enquanto o galho de injeção é transportado para terceiros externos, que fazem a reciclagem do PC, transformando-os em pellets para serem comercializados como matéria-prima de segunda linha. O fluxograma do processo, ou seja, as fronteiras do sistema, para o cenário 1 está representado na Figura 1.

O cenário 2 representa o processo que utiliza 20\% de PC virgem e $80 \%$ de PC reciclado para produzir as hastes plásticas. O reciclado (80\%) é proveniente dos "galhos de injeção" deste e de outros processos internos de produção da empresa (reciclagem interna). O virgem (20\%), proveniente do fornecedor, segue para a desumidificação e, em seguida, para a injeção, onde se une com a fração de $80 \%$ de PC reciclado internamente, formando a mistura de materiais virgens e reciclados que será utilizada para produção das hastes. 
Os produtos são separados manualmente, assim como no cenário 1 , porém os resíduos de $\mathrm{PC}$, ao invés de serem transportados para a reciclagem externa, seguem para um moinho. O equipamento já existente na fábrica é responsável pela reciclagem mecânica interna do PC, para sua futura reinserção no processo. A Figura 2 apresenta o fluxograma do processo considerado para o cenário 2 .

\section{Inventário do ciclo de vida}

Os dados necessários para o cálculo dos impactos ambientais foram coletados de acordo com as etapas existentes nos dois cenários, como mostrado nas Figuras 1 e 2. Essas etapas foram o transporte da matéria-prima virgem e a produção das hastes de PC utilizando os processos dentro da fábrica, como o desumidificador e a injetora, e, no caso do cenário 2, que utiliza PC reciclado, faz-se ainda necessária a adição de um moinho ao processo. Para o cenário 1, é necessário contabilizar também o transporte dos resíduos de PC até o terceiro externo, uma vez que, para esse cenário, esses resíduos não são reciclados na empresa. Todos os dados coletados foram normalizados conforme a unidade funcional, ou seja, "produção de um par de hastes".

Os dados de inventário para o cenário 1 são apresentados nas Tabelas 1 e 2. A Tabela 1 contém os dados de entrada e saída de material, com as quantidades de PC virgem utilizado e a de resíduos produzidos após a injeção, bem como o consumo de energia dos equipamentos usados (desumidificador e injetora) nesse cenário. Na Tabela 1, pode-se observar que, no cenário 1 , para produzir um par de hastes de óculos, são necessários 9,84 g de PC virgem e são gerados aproximadamente 2,57 g de resíduos de PC. Os dados do transporte (distância e quantidade transportada) de PC virgem até a empresa e do transporte dos resíduos de PC até o terceiro externo que ocorrem no cenário 1 são mostrados na Tabela 2 .

Os dados coletados para os fluxos de matéria e energia, assim como a etapa de transporte para o cenário 2, que utiliza 20\% de PC virgem e $80 \%$ de reciclado, são apresentados nas Tabelas 3 e 4 . A Tabela 3 mostra que, para esse cenário, são, então, utilizados apenas 1,97 g

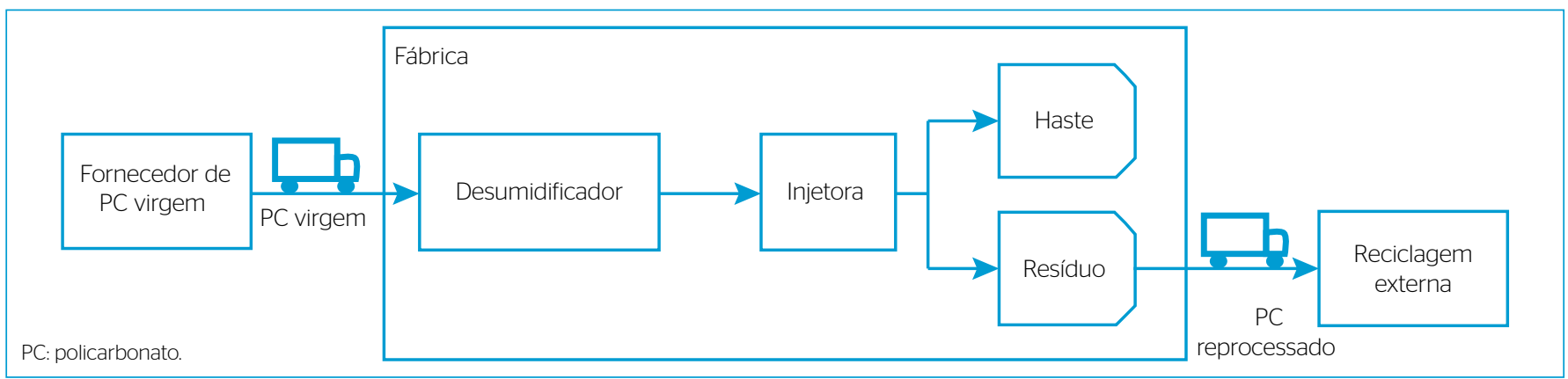

Figura 1 - Fluxograma do processo para o cenário 1.

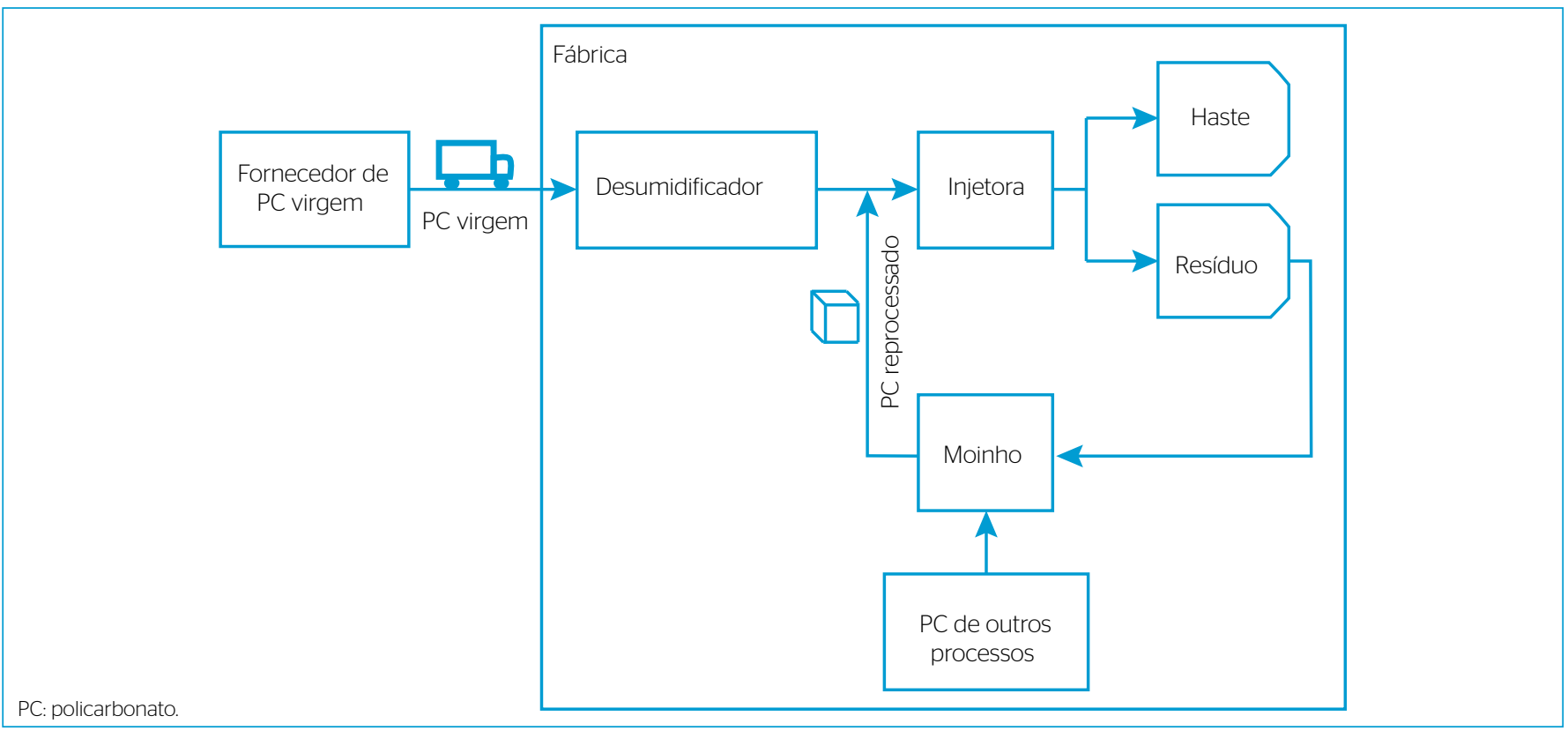

Figura 2 - Fluxograma do processo para o cenário 2. 
de PC virgem, sendo o restante 7,87 g de PC reciclado. O desumidificador passa agora a ser utilizado para secagem de somente $20 \%$ de PC virgem (Figura 2), o que justifica um consumo de energia de apenas 3,94 Wh para esse equipamento, como mostra a Tabela 3, valor bem

Tabela 1 - Inventário de materiais e consumo elétrico no cenário 1.

\begin{tabular}{c|c|c|c}
\multicolumn{4}{c}{ Cenário 1 - Fluxos de material e Energia } \\
\hline \multirow{3}{*}{ Dados } & Unidade & Consumo \\
\hline \multirow{3}{*}{ Entrada } & PC virgem & $\mathrm{g}$ & 9,84 \\
\cline { 2 - 4 } & Total & $\mathrm{g}$ & 9,84 \\
\hline \multirow{3}{*}{ Saída } & Produto final & $\mathrm{g}$ & 7,27 \\
\cline { 2 - 4 } & Resíduo & $\mathrm{g}$ & 2,57 \\
\cline { 2 - 4 } & Total & $\mathrm{g}$ & 9,84 \\
\hline \multirow{3}{*}{ Eletricidade } & Desumidificador & Wh & 16,90 \\
\cline { 2 - 4 } & Injetora & Wh & 79,40 \\
\cline { 2 - 4 } & Total & Wh & 96,30 \\
\hline
\end{tabular}

PC: policarbonato

Tabela 2 - Inventário da etapa de transporte para o cenário 1.

\begin{tabular}{l|c|c}
\multicolumn{3}{c}{ Cenário 1} \\
Dados & Unidade & Valor \\
Transporte PC virgem & $\mathrm{km}$ & 150 \\
\hline Distância & $\mathrm{g}$ & 9,84 \\
\hline Quantidade de PC & $\mathrm{km}$ & 187 \\
\hline Transporte de resíduos (até terceiro externo) & \\
\hline Distância & $\mathrm{g}$ & 2,57 \\
\hline Quantidade de PC
\end{tabular}

PC: policarbonato.

Tabela 3 - Inventário de materiais e consumo elétrico no cenário 2.

\begin{tabular}{l|c|c|c}
\multicolumn{4}{c}{ Cenário 2 - Fluxos de material e energia } \\
\hline \multirow{3}{*}{ Dados } & Unidade & Consumo \\
\hline \multirow{4}{*}{ Enterial } & PC virgem & $\mathrm{g}$ & 1,97 \\
\hline & PC reciclado & $\mathrm{g}$ & 7,87 \\
\cline { 2 - 4 } & Total & $\mathrm{g}$ & 9,84 \\
\cline { 2 - 4 } Saída & Produto final & $\mathrm{g}$ & 7,27 \\
\cline { 2 - 4 } & Resíduo de PC & $\mathrm{g}$ & 2,57 \\
\cline { 2 - 4 } & (vai para o moinho) & $\mathrm{g}$ & 9,84 \\
\hline \multirow{4}{*}{ Eletricidade } & Total & Wh & 3,94 \\
\cline { 2 - 4 } & Desumidificador & Wh & 79,40 \\
\cline { 2 - 4 } & Injetora & Wh & 4,60 \\
\cline { 2 - 4 } & Moinho & Wh & 87,40 \\
\hline
\end{tabular}

PC: policarbonato. menor que os 16,90 Wh de consumo do desumidificador para o cenário 1 (Tabela 1). Entretanto, no cenário 2, é preciso também considerar o consumo de energia do moinho, equipamento adicionado para realizar a reciclagem mecânica dos resíduos de PC. Embora o moinho represente um consumo adicional de $4,60 \mathrm{Wh}$, a energia total consumida pelo cenário 2 permanece menor que a do 1 . De acordo com a Tabela 3, o cenário 2 apresenta um consumo energético de 87,40 Wh, contra os 96,30 Wh do cenário 1.

A etapa de transporte do cenário 2 considera apenas o transporte de $20 \%$ de PC virgem do fornecedor até a empresa de fabricação dos óculos (Tabela 4). O transporte de resíduos até o terceiro externo não acontece nesse cenário, visto que os resíduos de PC são reciclados dentro da própria empresa.

\section{Avaliação dos impactos}

A avaliação dos impactos ambientais foi realizada utilizando o software SimaPro 8.2.3. Este é um dos softwares mais utilizados em estudos de $\mathrm{ACV}$, que apresenta uma grande quantidade de base de dados e diferentes métodos de avaliação de impacto. Os métodos de avaliação de impactos são responsáveis por converter os dados numéricos e os fluxos quantificados, na etapa de inventário, em resultados em termos de categorias de impactos. Esses métodos, também chamados de modelos de caracterização, contêm informações ambientais acerca de inúmeras substâncias, determinando, para cada uma delas, um fator de caracterização. A partir desses fatores de caracterização, alguns métodos oferecem ainda elementos opcionais de normalização e ponderação dos resultados, a fim de se adaptar a um contexto local e/ou social (RENOU, 2006).

O método de avaliação de impacto utilizado neste trabalho foi o ILCD 2011 Midpoint+, por ser um método de grande confiabilidade e sem aplicações específicas, adequado para várias situações e vários cenários. Esse método, que propõe 16 categorias de impactos, foi desenvolvido pela Comissão Europeia (EC-JRC-IES, 2011), que analisou diferentes métodos de avaliação de impactos e, a partir de uma primeira pré-seleção dos métodos existentes e da definição de critérios, estabeleceu uma nova lista de métodos recomendados para cada categoria de impacto.

\section{RESULTADOS E DISCUSSÃO}

Os dois cenários foram avaliados separadamente, com vistas a avaliar as contribuições de cada etapa do processo em termos de impactos

Tabela 4 - Inventário da etapa de transporte para o cenário 2.

\begin{tabular}{l|c|c|c}
\multicolumn{3}{c}{ Cenário 2} \\
\hline \multirow{2}{*}{ Dados } & Unidade & Valor \\
\hline \multirow{2}{*}{ Transporte } & Distância & $\mathrm{km}$ & 150 \\
\cline { 2 - 4 } PC virgem & Quantidade de PC & $\mathrm{kg}$ & 1,97 \\
\hline
\end{tabular}

PC: policarbonato. 
ambientais potenciais. Posteriormente, foram comparados, a fim de verificar qual é menos impactante ao meio ambiente.

Na Figura 3, tem-se a avaliação de impactos para o cenário 1 e é possível identificar a contribuição de cada atividade do processo, descrito na Figura 1, para cada 1 das 16 categorias de impactos ambientais analisadas por meio do método ILCD 2011 Midpoint+.

Analisando a Figura 3, é possível observar que a produção de PC virgem é a atividade responsável pela maior contribuição para os impactos ambientais referentes ao cenário 1, seguida pelo consumo elétrico dentro da fábrica. Essa produção é a atividade mais impactante em 11 das 16 categorias analisadas, sendo o consumo elétrico o mais relevante nas outras 5 .

Para as categorias de mudança climática (associada ao potencial de aquecimento global), toxicidade humana cancerígena (danosa aos tecidos dos organismos vivos) e material particulado (emitidos no ar e passíveis de inalação por seres vivos), a produção de PC virgem mostrou-se responsável por mais de $90 \%$ dos impactos ambientais nessas categorias.

Avaliando o consumo elétrico, a maior contribuição concentra-se na categoria radiação ionizada $\mathrm{HH}$ (tipos de radiação ionizada danosa à saúde humana), também superior a $90 \%$. Para o cenário 1 , a etapa de transporte apresenta uma baixa contribuição nos impactos ambientais, sendo as categorias de impactos que estão relacionadas ao consumo de combustíveis fósseis (relacionadas à escassez dos recursos naturais) e degradação da camada de ozônio (potencial de degradação dessa camada na atmosfera) as duas nas quais é possível observar uma contribuição mais significativa dessa atividade.

Na Figura 4, está apresentada a avaliação de impactos para o cenário 2. Essa avaliação mostra um maior equilíbrio entre os efeitos gerados pelo consumo elétrico e a produção de PC virgem, além de redução nos impactos causados pelo transporte de matéria-prima e a eliminação da necessidade de transportar resíduos até o terceiro externo.

A utilização de $80 \%$ de PC reciclado internamente acarreta a redução da produção de PC virgem, o que diminui os impactos causados por essa etapa. Por exemplo, a contribuição da produção de PC virgem para a categoria de mudanças climáticas, que antes era superior a $90 \%$, passa a ser de $70 \%$, ocasionando aumento na parcela de contribuição do consumo elétrico. Esse mesmo comportamento é observado para as outras categorias de impactos. No cenário 2, houve a necessidade de um equipamento a mais, o moinho, utilizado para a reciclagem interna do PC, resultando em um aumento no consumo elétrico da fábrica (4,60 Wh). Entretanto, a quantidade de PC que passa pelo desumidificador reduz-se a $20 \%$, diminuindo o consumo de energia elétrica para esse equipamento de $16,90 \mathrm{Wh}$, no cenário 1 , para 3,94 Wh, no 2 .

Portanto, o aumento da contribuição do consumo elétrico para os impactos ambientais no cenário 2, verificado na Figura 5, foi ocasionado pela redução da produção do PC virgem, uma vez que não

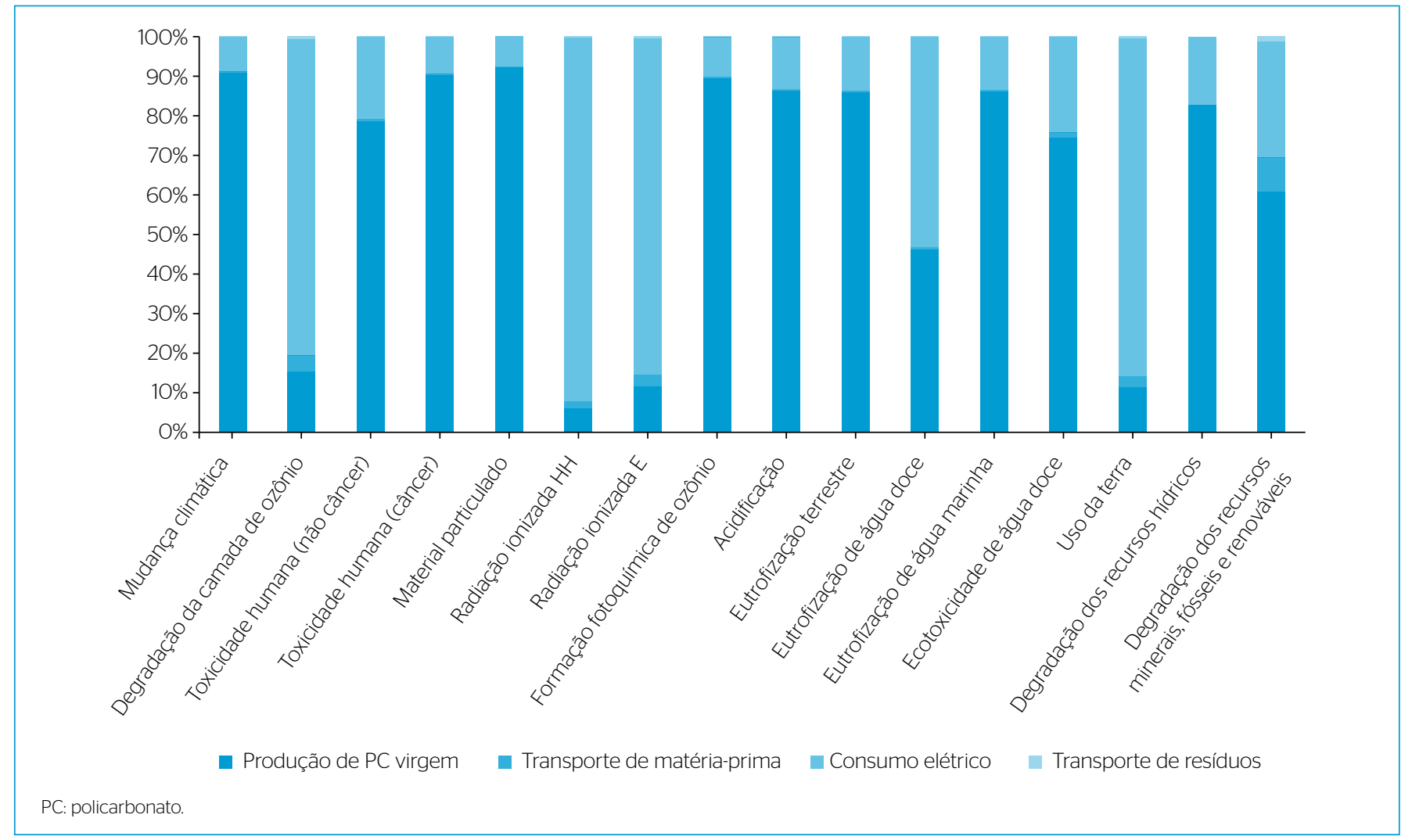

Figura 3 - Avaliação dos impactos ambientais para o cenário 1. 
houve aumento do consumo de energia elétrica, não provocando, assim, acréscimo dos impactos ambientais associados ao consumo elétrico.

Isso pode ser confirmado na Figura 5 e na Tabela 5, em que se tem a comparação dos impactos nos cenários 1 e 2, considerando todas as etapas desempenhadas no processo de produção de 1 par de hastes, permitindo identificar qual dos dois cenários é o menos impactante ao meio ambiente. Nelas, é possível observar que o cenário 2 é menos impactante ao meio ambiente do que o 1 para todas as categorias de impactos analisadas, apresentando reduções nos impactos superiores a 70\% em sete delas. As categorias de mudança climática e emissão de material particulado foram as mais afetadas, apresentando redução de quase $80 \%$ nos impactos ambientais quando se passa do cenário 1 ao 2 . Por outro lado, as categorias degradação da camada de ozônio, radiação ionizada $\mathrm{HH}$, radiação ionizada $\mathrm{E}$ (radiação danosa ao ecossistema) e uso da terra (uso de materiais orgânicos do solo) foram as que apresentaram as menores reduções de impactos para o cenário 2 em relação ao 1 .

Como observado anteriormente na Figura 3, a produção de PC virgem, matéria-prima para o processo estudado, foi a atividade que apresentou maior contribuição para a maioria dos impactos ambientais analisados e sua relevância pode ser confirmada após a comparação dos dois cenários na Figura 5, já que a redução da produção de PC virgem em $80 \%$ resultou em alterações expressivas nos impactos ambientais analisados. A incorporação do processo de reciclagem ao cenário 2 não representou grandes impactos para o meio ambiente, uma vez que se trata de um processo de reciclagem interna simples realizado por um equipamento de baixo consumo elétrico.

Resultados semelhantes foram obtidos no estudo de Kua e Lu (2016) para a substituição de vidro temperado por PC em janelas, pois a produção deste está atrelada a uma alta taxa de extração e utilização de combustíveis fósseis. Essa substituição foi também o fator de maior relevância nos impactos ambientais relacionados com a categoria de impacto mudança climática. A produção do PCé um processo complexo, que pode demandar uma quantidade considerável de recursos naturais e energia. Portanto, a reciclagem apresentou-se como alternativa importante para reduzir os impactos ambientais, uma vez que leva à diminuição de recursos naturais e matérias-primas usadas nos ciclos de vida dos produtos.

Verificou-se ainda que a quantidade de material reciclado utilizada poderia ser superior a $80 \%$, caso houvesse mais resíduos a serem reciclados, podendo atingir até $100 \%$ no processo em estudo. Isso se deve ao fato de as características do produto, nesse caso as hastes, permitirem a utilização de qualquer quantidade de material reciclado sem alterar sua funcionalidade. Em contrapartida, o material reciclado não poderia ser utilizado, por exemplo, na produção das lentes dos óculos, que possuem especificações menos flexíveis às alterações em sua composição, como a transparência e necessidade de suportar estresses mecânicos maiores.

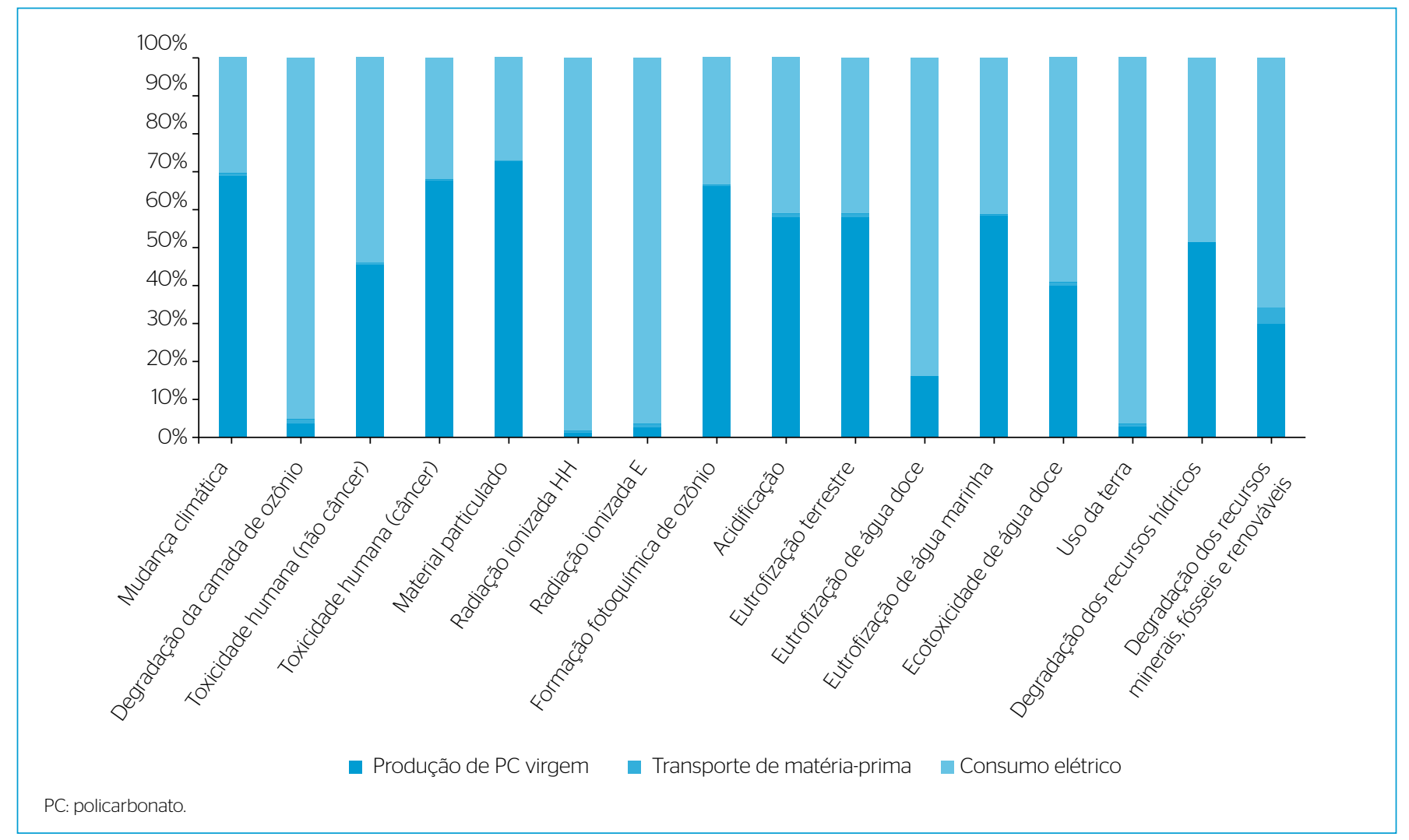

Figura 4 - Avaliação dos impactos ambientais para o cenário 2. 
Essas afirmações podem ser confirmadas pelo estudo de Kozderka et al. (2016), que compara o ciclo de vida do PP de alto impacto virgem e misturas de PP virgem com reciclado. Segundo o estudo, conforme a fração de material reciclado é aumentada, em relação ao material virgem, os impactos ambientais gerados decrescem, mostrando a eficácia ambiental da reciclagem do polímero para ser reaproveitado em misturas com sua versão virgem. Entretanto, as propriedades mecânicas, importantes para a funcionalidade do produto, são alteradas conforme a fração de PP reciclado é aumentada, impedindo a utilização de $100 \%$ de material reciclado dependendo da aplicação.

\section{CONCLUSÕES}

Os resultados da apreciação dos impactos ambientais avaliados mostraram uma redução considerável quando o processo de reciclagem interna, no cenário 2 (80\% PC reciclado - 20\% PC virgem), foi implementado. O transporte de resíduos apresentou uma contribuição mínima quando se analisa o cenário 1 (100\% PC virgem) comparando com as demais atividades existentes na fábrica. Por outro lado, a produção de PC virgem e o consumo de energia elétrica são os responsáveis pela grande maioria dos impactos ambientais avaliados nos dois cenários estudados.

Com a redução da utilização de matéria-prima virgem em $80 \%$, do cenário 1 para o 2, foi notável que a redução da produção de PC virgem teve uma grande influência na redução dos impactos ambientais,
Tabela 5 - Impactos ambientais dos cenários 1 e 2.

\begin{tabular}{|c|c|c|c|}
\hline \multicolumn{4}{|c|}{ Impactos na produção de um par de hastes } \\
\hline Categoria de impacto & Unidade & Cenário 1 & Cenário 2 \\
\hline Mudança climática & $\mathrm{kgCO}_{2} \mathrm{eq}$ & $1,70 \times 10^{-1}$ & $4,47 \times 10^{-2}$ \\
\hline $\begin{array}{l}\text { Degradação da camada } \\
\text { de ozônio }\end{array}$ & kgCFC-11 eq & $2,15 \times 10^{-9}$ & $1,64 \times 10^{-9}$ \\
\hline $\begin{array}{l}\text { Toxicidade humana, } \\
\text { não cancerígena }\end{array}$ & CTUn & $2,24 \times 10^{-8}$ & $7,74 \times 10^{-9}$ \\
\hline $\begin{array}{l}\text { Toxicidade humana, } \\
\text { cancerígena }\end{array}$ & CTUh & $5,49 \times 10^{-9}$ & $1,46 \times 10^{-9}$ \\
\hline Material particulado & $\mathrm{kgPM} 2,5 \mathrm{eq}$ & $1,71 \times 10^{-4}$ & $4,33 \times 10^{-5}$ \\
\hline Radiação ionizada HH & kBqU235 eq & $2,49 \times 10^{-3}$ & $2,12 \times 10^{-3}$ \\
\hline Radiação ionizada E & CTU e & $7,45 \times 10^{-9}$ & $5,96 \times 10^{-9}$ \\
\hline $\begin{array}{l}\text { Formação de camada } \\
\text { fotoquímica de ozônio }\end{array}$ & kgNMVOC eq & $4,13 \times 10^{-4}$ & $1,12 \times 10^{-4}$ \\
\hline Acidificação & molc $\mathrm{H}+\mathrm{eq}$ & $6,88 \times 10^{-4}$ & $2,03 \times 10^{-4}$ \\
\hline Eutrofização terrestre & molc N eq & $1,23 \times 10^{-3}$ & $3,65 \times 10^{-4}$ \\
\hline Eutrofização de água doce & $\mathrm{kgP}$ eq & $9,55 \times 10^{-6}$ & $5,51 \times 10^{-6}$ \\
\hline Eutrofização de água marinha & $\mathrm{kgNeq}$ & $1,13 \times 10^{-4}$ & $3,31 \times 10^{-5}$ \\
\hline Ecotoxicidade de água doce & CTU e & $3,67 \times 10^{-1}$ & $1,36 \times 10^{-1}$ \\
\hline Uso da terra & kgC déficit & $6,21 \times 10^{-2}$ & $4,98 \times 10^{-2}$ \\
\hline $\begin{array}{l}\text { Degradação de } \\
\text { recursos hídricos }\end{array}$ & mª́gua eq & $2,02 \times 10^{-4}$ & $6,52 \times 10^{-5}$ \\
\hline $\begin{array}{l}\text { Degradação de recursos } \\
\text { minerais, fósseis e renováveis }\end{array}$ & kgSb eq & $3,73 \times 10^{-7}$ & $1,51 \times 10^{-7}$ \\
\hline
\end{tabular}

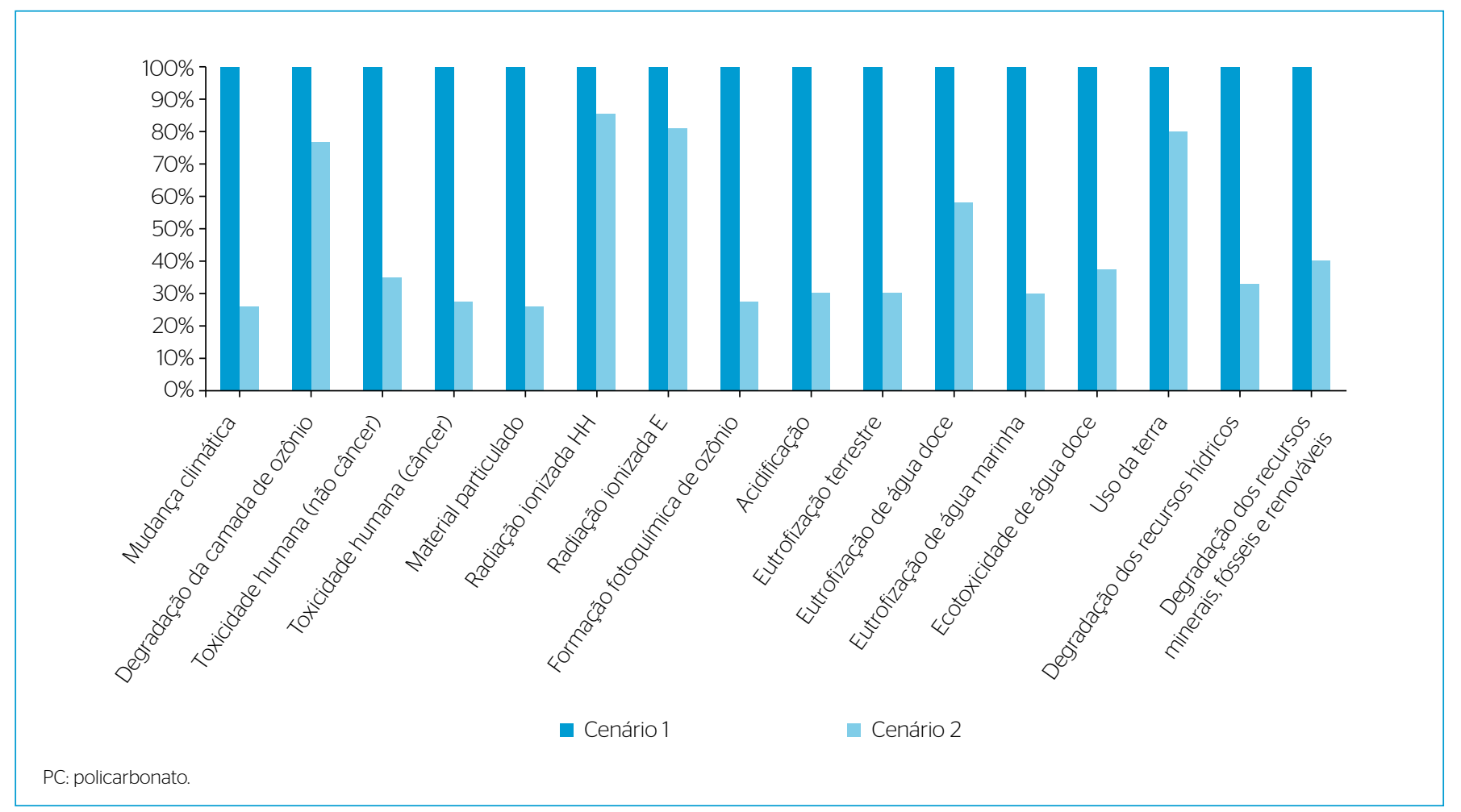

Figura 5 - Comparação dos impactos ambientais entre os cenários 1 e 2. 
mesmo com a necessidade de se utilizar o moinho para a reciclagem dos resíduos de PC. Esse fato é confirmado uma vez que o cenário 2 apresentou uma redução considerável em quase todas as categorias de impactos avaliados no presente trabalho. Em relação à emissão de $\mathrm{CO}_{2}$, foi calculado um valor igual a $1,7 \times 10^{-1} \mathrm{kgCO}_{2}$ eq para o cenário 1 e $4,47 \times 10^{-2} \mathrm{kgCO}_{2}$ eq para o 2 . Fato verificado graças ao atendimento das etapas propostas na metodologia do trabalho, o que foi imprescindível para a obtenção dos resultados finais.
Considerando apenas esses dados, os ganhos ambientais expressivos são conquistados com a adoção da reciclagem interna do $\mathrm{PC}$ no processo de produção das hastes, apresentando valores de reduções de impactos que chegam a mais de 70\%, como no caso da categoria de mudança climática. Os resultados encontrados e discutidos superaram as expectativas em relação à proporção das reduções apresentadas, mostrando que, em casos específicos, a reciclagem interna pode gerar resultados extremamente vantajosos para a empresa e para o meio ambiente.

\section{REFERÊNCIAS}

AL-SABAGH, A.M.; YEHIA, F.Z.; ESHAQ, G.; RABIE, A.M.; ELMETWALLY, A.E. (2016) Greener routes for recycling of polyethylene terephthalate. Egyptian Journal of Petroleum, v. 25, n. 1, p. 53-64. https://doi.org/10.1016/j.ejpe.2015.03.001

Campolina, J.M.; Sigrist, C.S.L.; Paiva, J.M.F.; Nunes, A.O.; Moris, V.A.S. (2017) A study on the environmental aspects of WEEE plastic recycling in a Brazilian company. International Journal Life Cycle Assessment, v. 22, p. 1957-1968. https://doi.org/10.1007/s11367-017-1282-2

CHEHEBE, J.R.B. (1997) Análise do ciclo de vida de produtos: ferramenta gerencial da ISO 1400O. Rio de Janeiro: Qualitymark. 104 p.

CURRAN, M.A. (2006) Report on Activity of Task Force 1 in the Life Cycle Inventory Programme: Data Registry - Global Life Cycle Inventory Data Resources. International Journal Life Cycle Assessment, v. 11, n. 4, p. 284-289.

DOS SANTOS, W.N.; AGNELLI, J.A.M.; MUMMERY, P.; WALLWORK, A. (2007) Effect of recycling on the termal properties of polymers. Polymer Testing, v. 26, n. 2, p. 216-221. http://dx.doi.org/10.1016/j. polymertesting.2006.10.004

EUROPEAN COMMISSION - JOINT RESEARCH CENTRE. (2O11) International Reference Life Cycle Data System (ILCD) Handbook - recommendations for life cycle impact assessment in the European context, 1st ed. EUR 24571 EN. Luxemburg: Publications Office of the European Union.

GU, L.; OZBAKKALOGLU, T. (2016) Use of recycled plastics in concrete: A critical review. Waste Management, v. 51, p. 19-42. https:// doi.org/10.1016/j.wasman.2016.03.005

GUTIÉRREZ, C.; RODRÍGUEZ, J.F.; GRACIA, I.; LUCAS, A. GARCÍA, M.T. (2016) Reduction of the carbon footprint through polystyrene recycling: economical evaluation. Process Safety and Environmental Protection, v. 101, p. 144-151. https://doi.org/10.1016/j.psep.2015.12.004

HAMAD, K.; KASEEM, M.; DERI, F. (2O13) Recycling of waste from polymer materials: An overview of the recent works. Polymer Degradation and Stability, v. 98, n. 12, p. 2801-2812. Recycling of waste from polymer materials: An overview of the recent works. Polymer Degradation and Stability
HOPEWELL, J.; DVORAK, R.; KOSIOR, E. (2009) Plastics recycling: challenges and opportunities. Philosophical Transactions of the Royal Society B, v. 364, n. 1526, p. 2115-2116. https://doi.org/10.1098/ rstb.2008.0311

HUNT, E.J.; ZHANG, C.; ANZALONE, N.; PEARCE, J.M. (2015) Polymer recycling codes for distributed manufacturing with 3-D printers. Resources, Conservation and Recycling, v. 97, p. 24-30. https://doi. org/10.1016/j.resconrec.2015.02.004

INTERNATIONAL ORGANIZATION FOR STANDARDIZATION (ISO). (2006) ISO 14040: Environmental management - Life cycle assessment-principles and framework. Genebra: ISO.

KOZDERKA, M.; ROSE, B.; KOCI, V.; CAILLAUD, E.; BAHLOULI, N. (2016) High impact polypropilene (HIPP) recycling - Mechanical resistance and life cycle assessment (LCA) case study with improved efficiency by preliminary sensitivity analysis. Journal of Cleaner Production, v. 137, p. 1004-1017. https://doi.org/10.1016/j. jclepro.2016.07.217

KUA, H.W.; LU, Y. (2O16) Environmental impacts of substituting tempered glass with polycarbonate in construction - A attributional and consequintial life cycle perspective. Journal of Cleaner Production, v. 137, p. 910-921. http://dx.doi.org/10.1016/j.jclepro.2016.07.171

LINO, F.A.M.; ISMAIL, K.A.R.; COSSO, I.L. (2013) Evaluation of the potential of recycling for the reduction of energy and $\mathrm{CO} 2$ emissions in Brazil. Sustainable Cities and Society, Campinas, v. 8, p. 24-30. https://doi.org/10.1016/j.scs.2012.12.002

MADAN,J.; MANI, M.;LEE,J.H.; LYONS, K.W.(2O15)Energyperformance evaluation and improvement of unit-manufacturing processes: injection molding case study. Journal of Cleaner Production, v. 105, p. 157-170. https://doi.org/10.1016/j.jclepro.2014.09.060

MERRINGTON, A. (2011) Recycling of Plastics. In: KUTZ, M. (org.). Applied plastics engineering handbook - Processing, Materials and Applications. Reino Unido: Elsevier. p. 177-191.

PERCHENART, E.; ROQUESALANE, A. (2014) SimaPro: Iogiciel d'analyse de cycle de vie. In: TECHNIQUES DE L'INGÉNIEUR. França, $20 \mathrm{p}$. 
PlasticsEurope. (2011) Eco-profiles and Environmental Declarations: PlasticEurope Version 2.O. Eco-profiles and Environmental Product Declarations of the European Plastics Manufacturers PlasticsEurope. Bruxelas: PlasticsEurope. 50 p.

RENOU, S. (2006) Analyse de cycle de vie appliquée aux systèmes de traitement des eaux usées. Tese (Doutorado) - Institut National Polytechnique de Lorraine, França.

SAVAGE, J.; HARTLEY, Q. (2O10) Reprocessing of polymeric materials for reincorporation into moulded components. Sealing Technology, v. 2010, v. 3, p. 8-11. https://doi.org/10.1016/S1350-4789(10)70140-9

SINGH, N.; HUI, D.; SINGH, R.; AHUJA, I.P.S.; FEO, L.; FRATERNALI, F. (2016) Recycling of plastic solid waste: A state of art review and future applications. Composites Part B, v. 115, p. 409-422. https://doi. org/10.1016/j.compositesb.2016.09.013

SOCIETY OF ENVIRONMENTAL TOXICOLOGY AND CHEMISTRY (SETAC). (1993) Guidelines for Life-Cycle Assessment: A Code of Practice. Bruxelas: SETAC.

SPINACÉ, M.A.S.; PAOLI, M.A. (2005) A tecnologia de reciclagem de polímeros. Química Nova, v. 28, n. 1, p. 65-72.
UNITED STATES ENVIRONMENTAL PROTECTION AGENCY (USEPA). LCA Resources. Disponível em: <https://www.epa.gov/>. Acesso em: jan. 2017.

WANG, C.; WANG, H.; FU, J.; LIU, Y. (2015) Flotation separation of waste plastics for recycling - A review. Waste Management, v. 41, p. 28-38. https://doi.org/10.1016/j.wasman.2015.03.027

WITIK, R.A.; TEUSCHER, R.; MICHAUD, V.; LUDWIG, C.; MANSON, J.A.E. (2013) Carbon fibre reinforced composite waste: an environmental assessment of recycling, energy recovery and landfilling. Composites Part A, v. 49, p. 89-99. http://dx.doi. org/10.1016/j.compositesa.2013.02.009

Zanghelini, G.M.; SOUZA JÚNIOR, H.R.A.; CHERUBINI, E.; KULAY, L.; SOARES, S.R. (2016) Análise da evolução dos temas de pesquisa da ACV no Brasil baseada na relação de co-words. LALCA: Revista Latino Americana em Avaliação do Ciclo de Vida, p. 34-47.

Zhu, P.; Liu, X.; Wang, Y.W.; Guan, C.J.; Yang, Y.H.; Zhu, J.; Li, X.; Qian, G.; Frost, R.L. (2017) Production and characterization of recycled polycarbonate based composite material containing recycled glass fibers. Journal of Environmental Chemical Engineering, v. 5, n. 4, p. 3539-3446. http://dx.doi.org/10.1016/j.jece.2017.06.050 